\title{
Magneto-Plasmonic Nanoparticle Grid Biosensor with En- hanced Raman Scattering and Electrochemical Transduction for Development of Nanocarriers for Targeted Delivery of Pro- tected Anticancer Drugs
}

\author{
Hoda Ilkhani ${ }^{1,2, *}$, Chuan Jian Zhong ${ }^{3}$, and Maria Hepel ${ }^{1, *}$ \\ 1 Department of Chemistry, State University of New York at Potsdam, Potsdam, NY 13676, USA; he- \\ pelmr@potsdam.edu \\ 2 Central New Mexico Community College, Albuquerque, NM 87106, USA; hodailkhani@gmail.com \\ 3 Department of Chemistry, Binghamton University, Potsdam, Binghamton, NY 13676, USA; cjzhong@bing- \\ hamton.edu \\ * Correspondence: hepelmr@potsdam.edu; Tel.: 001.315.26727 (MH); hodailkhani@gmail.com (HI)
}

\begin{abstract}
Safe administration of highly cytotoxic chemotherapeutic drugs is a challenging problem in cancer treatment due to the adverse side effects and collateral damage to non-tumorigenic cells. To mitigate these problems, new promising approaches, based on the paradigm of controlled targeted drug delivery (TDD), utilizing drug nanocarriers with biorecognition ability to selectively target neoplastic cells, are being considered in cancer therapy. Herein, we report on the design and testing of a nanoparticle-grid based biosensing platform to aid in the development of new targeted drug nanocarriers. The proposed sensor grid consists of superparamagnetic gold-coated core-shell $\mathrm{Fe}_{2} \mathrm{Ni} @ \mathrm{Au}$ nanoparticles, further functionalized with folic acid targeting ligand, model thiolated chemotherapeutic drug doxorubicin (DOX), and a biocompatibility agent, 3,6,-dioxa-octanethiol (DOOT). The employed dual transduction based on electrochemical and enhanced Raman scattering detection have enabled efficient monitoring of the drug loading onto the nanocarriers, attached to the sensor surface, as well as the drug release under simulated intracellular conditions. The grid's nanoparticles serve here as the model nanocarriers for new TDD systems under design and optimization. The superparamagnetic properties of the Fe2Ni@Au NPs aid in nanoparticles' handling and constructing a dense sensor grid with high plasmonic enhancement of the Raman signals due to the minimal interparticle distance.
\end{abstract}

Keywords: Raman biosensor; Au-coated nanoparticles; hot-spot SERS substrate; anticancer drug nanocarriers; targeted drug delivery sensing; magneto-plasmonic nanoparticles.

\section{Introduction}

With the new advances in nanobiotechnology, the targeted drug delivery (TDD) of cytotoxic chemotherapeutics is now becoming one of the most promising approaches in cancer treatment [1-3]. The TDD systems are based on drug nanocarriers, equipped with biorecognition capability directed toward cancer cells [4,5]. Further interests in TDDs arise from their ability to mitigate both the adverse side effects of drugs and the collateral damage to non-tumorigenic cells, which cannot be easily achieved with a systemic drug administration used in classical chemotherapy. Moreover, there are indications that a TDD may protect the drugs against inactivation by the biological medium and likely alleviate the multidrug resistance of tumors [6]. In this work, we have investigated new platforms for designing and testing of drug nanocarriers for applications in new TDD systems. The proposed novel biosensing platform is based on a monolayer of model nanocarriers immobilized on a $\mathrm{Au}(111)$ substrate or a gold disk electrode (AuDE). The nanocarriers consist of superparamagnetic gold-coated $\mathrm{Fe}_{2} \mathrm{Ni} @ \mathrm{Au}$ core-shell nanoparticles, functionalized with a chemotherapeutic drug doxorubicin (DOX) and other functional molecules. For sensitive monitoring of analytical signals associated with drug loading and releasing, a 
dual surface-enhanced Raman light scattering (SERS) and electrochemical transduction has been employed. The proposed biosensing platform may serve for the development of new nanodrugs and for studies of controlled drug release from nanocarriers under simulated intracellular conditions.

Chemotherapy has widely been recognized as one of the more successful methods in cancer therapy. The classical chemotherapy, based on a systemic administration of highly cytotoxic drugs, is able to preferentially kill cancer cells. Unfortunately, this therapy has serious disadvantages, such as the severe side effects, high collateral damage to healthy cells and it may lead, in some cases, to the organ failure or systemic collapse. Therefore, novel approaches have now been aimed at replacing classical chemotherapy with nanotechnology-driven theranostic methods that can mitigate its drawbacks. The new approaches are based on a targeted delivery of anticancer drugs directly to cancer cells using specially designed drug nanocarriers.

A variety of nanoparticles (NPs) have been designed and investigated for applications as the nanocarriers in TDD, including liposomes [7], solid lipid NPs [8], empty-shell biomolecules, such as apoferritin [9] or cyclodextrin [10], biodegradable particles [11], polymeric dendrimer nanostructures [4], plasmonic [12,13] and magnetic [14] NPs, and others.

The immobilization of drugs, targeting ligands and other functional molecules onto the nanoparticle nanocarriers involves various kinds of binding, including electrostatic interactions [15,16], covalent binding [12-14,17-19], adsorption [20,21], supramolecular interactions [9,22], and trapping [7]. The drug binding to nanocarriers and encapsulation processes have been carried out to improve pharmacological and therapeutic effects of the drugs in terms of reducing collateral damage and side effects [23-25], while increasing the local drug potency at the targeted cancer cells. The modality of drug binding to nanocarriers has to be carefully assessed taking also into account the specificity of the subsequent controlled drug release processes at the target tumor cells [26-28].

The drug-loaded nanocarriers themselves require protection against damage due to the immune system response and opsonization [29-31]. In order to attain the proper biocompatibility, a nanocarrier is ordinarily functionalized by immobilization of pegylated ligands on its surface [29,32]. Biocompatible polyelectrolyte complex nanostructures and NPs with other coatings can also be designed to serve as the TDD nanocarriers [31,33]. On the other hand, the biogenic nanocarriers, such as those based on apoferritin nanocage carriers [9], generally, do not require any extra biocompatibility ligands.

Doxorubicin, used in this work as the model chemotherapeutic drug, is one of the most widely used anticancer drugs [34]. It is especially effective in the treatment of breast cancer, lymphoma, leukemia, bladder cancer, Kaposi's sarcoma, and others. Upon an entry into a cell, DOX molecules follow to the nucleus, intercalate into the DNA duplex and act against topoisomerase II, an enzyme active in DNA replication and repair. The DOX intercalation results in DNA cleavage [35]. There are several disadvantages of cancer treatment with DOX, including high toxicity to healthy cells, serious side effects, low retention of the drug, and low solubility in aqueous solutions. The side effects include hair loss, vomiting, rash, and mouth inflammation. Also, the cardiotoxicity and myelosuppression, due to DOX, are the results of a local toxicity, occurring at the regular drug doses [36]. In our recent study, to mitigate the adverse side effects of a chemotherapeutic drug gemcitabine, we have applied binding of the drug molecule to a nanocarrier via its most active functional group [13]. Such binding deactivates the drug while it is transported on the nanocarrier through a biological environment. Therefore, in this work, DOX was bound to a linker, mercaptopropionic acid (MPA), immobilized on gold nanoparticles (GNPs) or magnetic nanoparticles (MNPs) by its $-\mathrm{NH}_{2}$ functional group. It has been demonstrated that this kind of an approach enables to increase the local drug dosing at the target cancer cells, thus improving the treatment efficacy [37].

Folic acid receptors (FRs), found to be overexpressed in different types of cancer cells including breast cancer [4], serve as the main targeted receptors, specific to the cancer cells. Normal cells, in contrast, express only a very small number of FRs, localizing them 
on the apical surface of polarized epithelia, where the receptors remain inaccessible to the drugs [38]. Hence, the nanocarriers with folate conjugates can selectively interact with cancer cells, by recognizing the overexpressed FRs, and be internalized in cancer cells via the receptor-mediated endocytosis. By this mechanism, folic acid-covered and drugloaded nanocarriers can overcome drug resistance caused by P-glycoprotein efflux pumps [39]. Thus, the availability of folic acid receptors in cancer cell membrane makes them very efficient agents for drug targeting [40].

For the detection of subtle changes in surface concentrations of active compounds, surface-enhanced Raman spectroscopy (SERS) is often utilized due its high sensitivity and functional groups' recognition capability. In previous works, we have employed SERS for the analysis of DNA damage caused by strong oxidants and drugs [18] and for monitoring of chemotherapeutic drug upload and release from the nanoparticle nanocarriers [12,13,41-43]. The quantitative Raman assays have also been developed for various solid drug dosing formats, such as capsules, tablets, and powders [44-48]. In addition, Gotter et al. [49] have described the use of Raman spectroscopy for the quantification of a drug suspended in a simple semi-solid formulation consisting of paraffin. More complex solid pharmaceutical formulations were analyzed by Hargreaves et al. [50], who established Raman spectroscopy for quantitative analysis of multi-component pharmaceutical capsules. Furthermore, Stillhart et al. [51] have demonstrated the method suitability for the quantification of three low-level excipients in the formulation. Raman spectroscopy is very useful in drug analysis not only due to the high sensitivity and chemical specificity, but also due to the ease of use, minimal sample handling, and significant discrimination against packaging materials and tablet excipients [52]. The addition of electrochemical sensing to the SERS monitoring enables convenient characterization of the properties of nanocarriers since voltammetric techniques offer high sensitivity and scalability, and can be readily integrated in various types of biosensors [12,18,53]. Recently, a variety of SERS substrates with high signal amplifications due to overlapping plasmonic fields have been proposed for biosensor applications, including localized surface plasmons' (LSPs) coupling that creates the enhancement "hot spots" effect for the Raman scattering intensity $[12,18,41,54,55]$, as well as coupling of multiple plasmon modes [56,57], including LSPs, surface plasmon polaritons (SPPs) and bulk plasmon polaritons (BPPs). For very dilute analytes and trace concentrations, various kinds of molecular enrichment, including capillary action with solvent evaporation, magnetic separation, molecular imprinting, and others, were recently applied [58,59].

By using magnetic nanoparticle-based drug nanocarriers and the external magnetic field focusing, it becomes now possible to directly move and localize drugs to solid tumors to reduce the side effects of anticancer drugs [35,60,61]. Magnetic nanoparticles offer the benefit of utilizing both the enhanced permeability and retention (EPR) effect (referred to as the passive targeting) whilst also ensuring a direct, guided delivery to the tumor (active targeting) [61-63]. Another advantage of this nanotechnology is the enhancement of the magnetic resonance imaging and induction of cytotoxicity through the near-infrared derived hyperthermia [64,65]. Unfortunately, many magnetic nanoparticles, such as the superparamagnetic iron oxide, $\mathrm{Fe}_{3} \mathrm{O}_{4}$ (magnetite), are unstable without protection and in physiological media undergo degradation due to oxidation, aggregation, and precipitation. Also, the functionalization of non-metallic magnetic NPs is challenging $[63,66]$. In vivo, this may result in an early release of anticancer drug whilst still in the blood stream and failure of the drug to reach the tumor [38]. Therefore, to address these problems, we and others have developed new magnetic-alloy carriers, such as the superparamagnetic $\mathrm{Fe}_{2} \mathrm{Ni} @ \mathrm{Au}$ core-shell nanocarriers $[14,18,41,67]$. The Au shell provides a convenient platform for the surface functionalization of the NPs. Different kinds of macromolecules, such as peptides [39] and polymers, [40,68] or hydrophilic ligands forming a self-assembled monolayer (SAM), [66,69-71] can be utilized for this purpose.

Herein, we report on the development of a new magneto-plasmonic grid sensor with electrochemical and surface-enhanced Raman scattering (SERS) transduction enabling to investigate drug nanocarriers, including functionalization with targeting ligands, 
attachment of biocompatibility enhancing molecules, and monitoring of drug uploading and release. The SERS sensor is based on a $\mathrm{Au}(111)$ substrate with a monolayer of goldcoated core-shell magneto-plasmonic nanocarriers $\mathrm{Fe}_{2} \mathrm{Ni} @ \mathrm{Au}$ with inter-particle plasmonic field "hot-spot" enhancement. The utility of the proposed methodology is demonstrated for the case of the development of a targeted delivery of a model anticancer drug doxorubicin (DOX), folic acid targeting ligand, and 3,6-dioxa-octanethiol biocompatibility agent onto the magneto-plasmonic nanoparticle nanocarriers (MPNP@Au). All steps of the nanocarriers synthesis and testing are described in detail.

\section{Materials and Methods}

\subsection{Materials}

The anticancer drug doxorubicin (DOX) used in this work was received from Selleck Chemicals (Houston, Texas, U.S.A.). Chemical compounds used for functionalization of nanocarriers and linking, including 1-octanethiol (OT), 1,6-hexanedithiol (HDT), 3,6-dioxa-octanethiol (DOOT), 3,6-dioxa-1,8-octanedithiol (DOODT), folic acid (FA), cysteamine hydrochloride (CYS), 5-phenyl-1,3,4-oxadiazole-2-thiol (PODAT), and 4-aminothiophenol (PATP), were acquired from Sigma-Aldrich Company (Milwaukee, Wisconsin, U.S.A.). Covalent binding of functional ligands was achieved using N-(3-dimethylaminopropyl)-N'-ethylcarbodiimide hydrochloride (EDC) and N-hydroxysuccinimide (NHS) to form an amide bonding. All reagents were of the analytical grade and were used as received. The reduced graphene oxide nanosheets (rGO) were obtained by oxidation of graphite flakes in acidic permanganate solution at $50{ }^{\circ} \mathrm{C}$, followed by partial electrochemical reduction, using a method we have recently developed for synthesis of electrocatalysts for polyphenol sensors [20]. The Au(111) gold substrates (250 nm thick, deposited on a borosilicate glass coated with a $4 \mathrm{~nm} \mathrm{Cr}$ adhesion layer) were obtained from Arrandee ${ }^{\mathrm{TM}}$ company (Werther, Germany). Spherical gold nanoparticles (5 to $100 \mathrm{~nm}$ dia.) were purchased from Nanopartz Inc. (Loveland, Colorado, U.S.A.). The magneto-plasmonic core-shell nanoparticles, $\mathrm{Fe} 2 \mathrm{Ni} @ \mathrm{Au}$, were synthesized and tested according to the procedure published earlier [67]. All aqueous solutions were prepared using a deionized water with $18.2 \mathrm{M} \Omega \mathrm{cm}$ resistivity, purified with a Millipore purification system (Bedford, Massachusetts, U.S.A.).

\subsection{Modification of $A u(111)$ substrates with nanocarrier grids}

The sensor substrates made of a $\mathrm{Au}(111)$ film were cleaned by etching in freshly made piranha solution (3:1 v/v concentrated $\mathrm{H}_{2} \mathrm{SO}_{4}: 30 \% \mathrm{H}_{2} \mathrm{O}_{2} ;$ CAUTION: Piranha solution is very dangerous, corrosive, and may explode if contained in a closed vessel; it should be handled with special care). After 10 min etching, the substrates were thoroughly rinsed with Milli$\mathrm{Q}$ water. To form a self-assembled monolayer (SAM) of a dithiol linker, a cleaned substrate was immersed in absolute ethanol solution containing $1.0 \mathrm{mM}$ 1,6-hexanedithiol (HDT) and $10 \mathrm{mM}$ 1-octanethiol (OT) (as diluent during the self-assembly process, to prevent forming bridged binding of HDT), with a volume ratio of 1:1, for $20 \mathrm{~h}$. After the self-assembly process, Au sensors were thoroughly rinsed with ethanol and water to remove physically adsorbed HDT and OT and then dipped into a solution of gold nanoparticles (GNPs) or magnetic nanoparticles (MNPs), for $3.5 \mathrm{~h}$ to form a monolayer grid of plasmonic NPs. The obtained nanoparticle grid was suitable for SERS measurements, as well as for the electrochemical characterization. The obtained nanoparticle grid-modified $\mathrm{Au}(111)$ sensors were washed with deionized water and used for further functionalization with protected anticancer drug (pDOX), folate targeting ligand (FTL), and biocompatibility agent (DOOT). The steps leading to the immobilization of GNPs and MNPs on Au substrates and nanoparticles' functionalization are illustrated in Scheme 1.

\subsection{Doxorubicin loading onto GNP and MNP nanocarriers}


Since the anticancer drug doxorubicin has a free $\mathrm{NH}_{2}$ group, it can be readily attached to the nanocarriers via linkers with carboxyl groups. For Au-coated MNPs and GNPs, the use of mercapto-propionic acid (MPA) as the linker is the proper choice. It forms a welldefined SAM on Au-surface with strong thiolate bonding. Thus, the nano-grid electrodes, $\mathrm{Au}(111) @ \mathrm{HDT} / \mathrm{MNP}$ and $\mathrm{Au}(111) @ \mathrm{HDT} / \mathrm{GNP}$, were soaked in $10 \mathrm{mM}$ MPA solution for 4 $\mathrm{h}$ to form a linker SAM, followed by thorough washing with distilled water. The surface carboxyl groups of MPA were then activated by EDC/NHS coupling in DMSO medium for $1 \mathrm{~h}$ and subsequently, a $0.5 \mathrm{mg} / \mathrm{mL}$ DOX solution in DMSO was added to complete the drug binding to the functionalized nanocarrier. After incubating for $4 \mathrm{~h}$, the electrode was rinsed with DMSO and 3 times with water and saline phosphate buffer, pH 7.4.

(A)
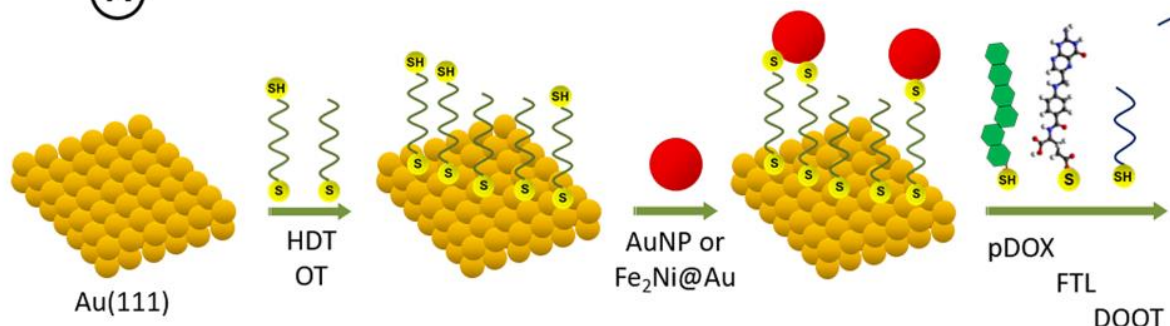

TL

DOOT

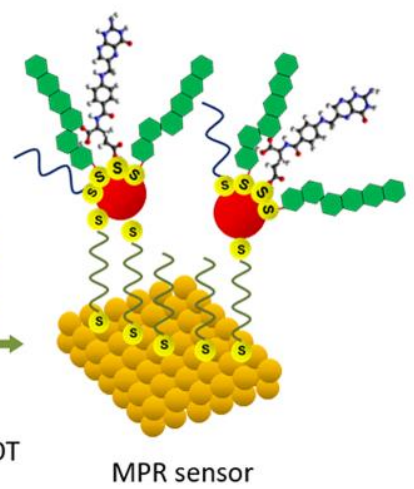

(B)

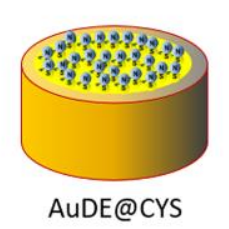

$\underset{\text { rGO }}{\stackrel{2}{\longrightarrow}}$
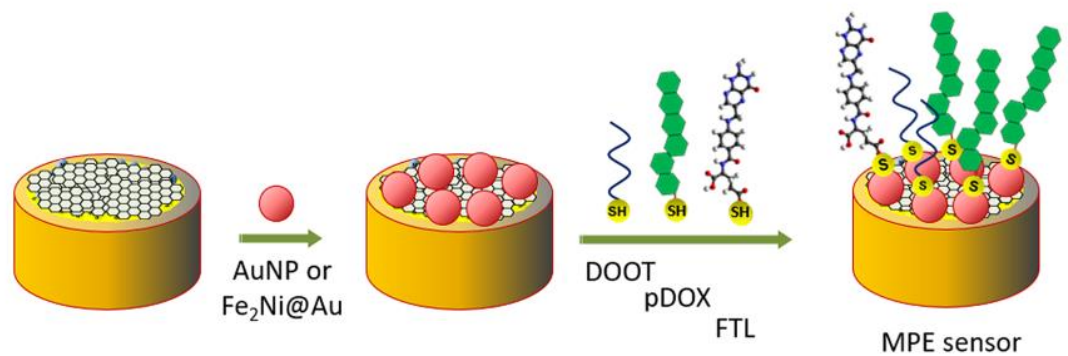

$\sum_{\text {HDT }}^{\text {SH }}$

$\sum_{\text {SH }}$
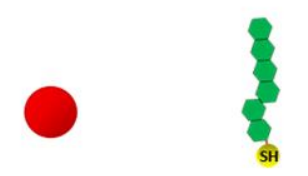

AuNP or

$\mathrm{Fe}_{2} \mathrm{Ni} @ \mathrm{Au}$

pDOX

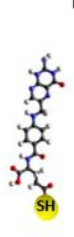

FTL

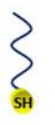

DOOT

Scheme 1. Construction of SERS-electrochemical sensors for development of targeted drug nanocarriers. HDT - hexanedithiol, OT - octanethiol, pDOX - protected thiolated anticancer drug doxorubicin, FTL - thiolated folate targeting ligand, DOOT - biocompatibility agent 3,6,-dioxaoctanethiol.

\subsection{Simultaneous functionalization of nanocarriers with anticancer drug (DOX), targeting} ligand (FA), and biocompatibility agent (DOOT)

Simultaneous functionalization of free nanocarriers, as well as the nanocarriers bound to nanogrid probes, with anticancer drugs, targeting ligands, and biocompatibility agents was carried out using pre-thiolated compounds. Thus, doxorubicin was first coupled with MPA via EDC/NHS amide bond formation, as shown in Scheme 2. 


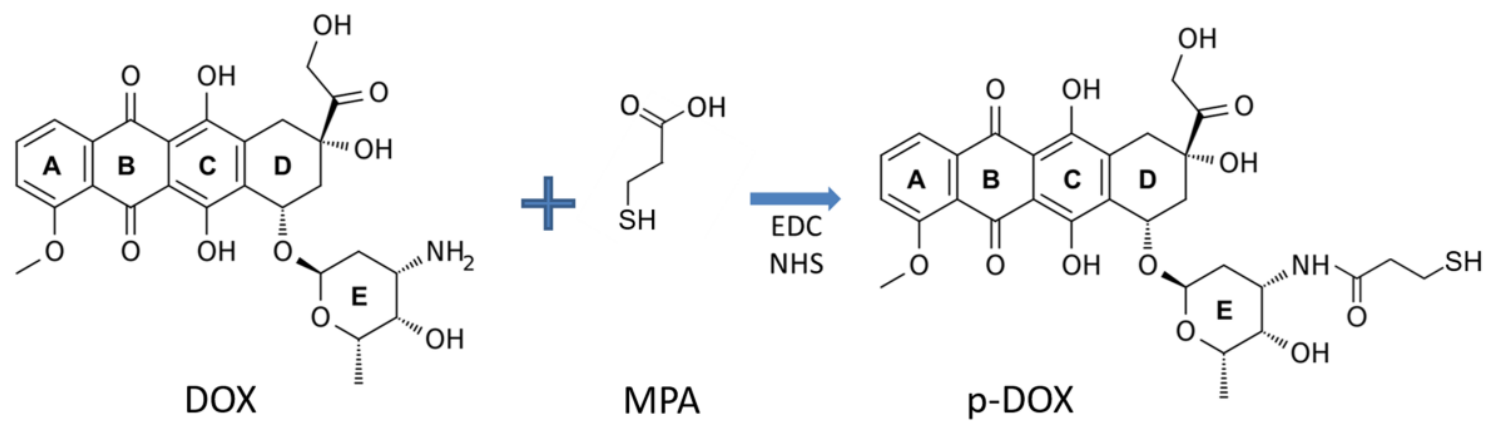

Scheme 2. Binding of MPA linker to doxorubicin via EDC/NHS-mediated amide bond formation process to protect the active $\mathrm{NH}_{2}$ group of the drug and provide a sulfhydryl moiety needed for attaching of the drug to Au-coated MNPs.

Folic acid targeting ligand, as the second component of the functionalizing solution, was obtained by activating FA with EDC/NHS, followed by bonding to cysteamine (CYS). The biocompatibility agent, 3,6,-dioxa-octanethiol (DOOT), in concentration $20 \mu \mathrm{g} / \mathrm{mL}$, was used as the third component in the functionalization solution. The nanogrid probes were dipped into the functionalization solution with component ratio: pDOX : FTL : DOOT $=20: 1: 1$ and incubated for $1 \mathrm{~h}$. The probes were thoroughly rinsed with PBS buffer saline $\mathrm{pH}$ 7.4. A model MNP-based drug nanocarrier functionalized with $\mathrm{pDOX}$, FTL and DOOT is depicted in Scheme 3.

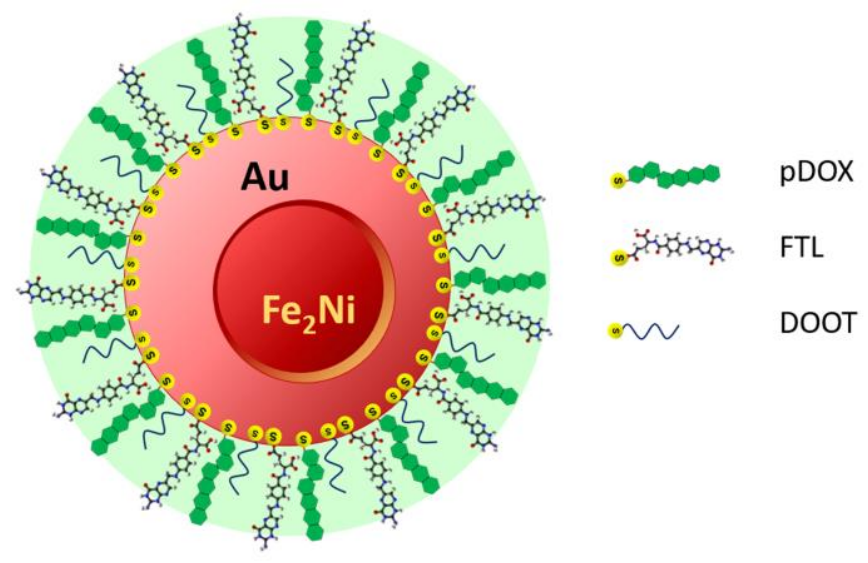

Scheme 3. Magneto-plasmonic nanocarrier with $\mathrm{Fe}_{2} \mathrm{Ni}$ core and Au shell coated with folate derivative as the folate targeting ligand (FTL), ethylene glycol derivative (DOOT) as the biocompatibility agent, and protected doxorubicin (p-DOX) as the chemotherapeutic drug.

\subsection{Polycrystalline gold disk electrode modification for electrochemical detection}

The modification of polycrystalline solid disk electrode (AuDE) for loading of doxorubicin was similar to that published previously for investigations of the damage to DNA caused by chemotherapeutics [18]. Briefly, the gold disk electrode was cleaned, after polishing with alumina powder $(0.05 \mu \mathrm{m}$ dia.), by potential scanning in a potential window between -0.4 and $1.7 \mathrm{~V}$ vs. SCE in $0.5 \mathrm{M} \mathrm{H}_{2} \mathrm{SO}_{4}$ at scan rate $\mathrm{v}=100 \mathrm{mV} / \mathrm{s}$, until the cyclic voltammograms for a clean Au electrode were obtained. The electrode was then modified with cysteamine $\mathrm{HCl}$ (CYS) as a linker for the attachment of graphene oxide (GO) nanosheets. The Au electrode was immersed in a $5 \mathrm{mM}$ CYS solution for $30 \mathrm{~min}$. After washing with distilled water, the AuDE@CYS electrode was immersed in an aqueous solution of GO with added EDC/NHS coupling agents for $4 \mathrm{~h}$ to attach single layer GO sheets to the CYS linker by amide bonding with carboxyl groups of GO. The obtained AuDE@CYS/GO electrode was then washed in PBS buffer and distilled water. Next, the AuDE@CYS/GO electrode was activated with EDC/NHS coupling agents for $4 \mathrm{~h}$. The CYS- 
capped MNPs (or GNPs) were then added to bind these nanoparticles to the AuDE/CYS/GO electrode surface. After incubation for $1 \mathrm{~h}$, the electrode was rinsed with water and PBS buffer pH 7.4. The obtained AuDE@CYS/GO/MNPs (or AuDE@CYS/GO/AuNPs) electrode was then scanned in $0.5 \mathrm{M} \mathrm{NaCl}$ solution from 0.7 to $-1.1 \mathrm{~V}$ at a scan rate of $50 \mathrm{mV} / \mathrm{s}$ to reduce the $\mathrm{GO}$ and form a reduced graphene oxide (rGO) film [72]. Thus, the AuDE/CYS/rGO/MNPs and AuDE/CYS/rGO/AuNPs electrodes were obtained. They were then loaded with an anticancer drug or mixture of ligands to complete the immobilized-nanocarrier functionalization, described in sections above.

\section{6. $p H$-responsive release of doxorubicin from nanocarriers}

The stimulated drug release from the surface of bound MNPs in Au(111)@HDT/MNPs@MPA/DOX sensors was monitored in solutions of different pH: in PBS at pH 7.4 and in acetate buffer solutions at pH 6.5, 5.3 and 4.5, using SERS measurements after different release times. The doxorubicin release was also investigated using voltammetric methods, CV and DPV, with AuDE@CYS/rGO/MNP@MPA/DOX electrode.

\section{Results and Discussion}

In this work, magnetic $\mathrm{Fe}_{2} \mathrm{Ni} @ \mathrm{Au}$ nanoparticles (MNPs) were synthesized and functionalized with an anticancer drug doxorubicin (DOX) and folic acid (FA) as the targeting ligand. A biocompatibility agent, 3,6-dioxa-octanethiol (DOOT), was also attached to MNPs to prevent the organism immunoresponse. The drug loading onto the nanocarriers was closely monitored with surface-enhanced RAMAN scattering (SERS) and electrochemical relaxation methods: cyclic voltammetry $(\mathrm{CV})$ and differential-pulse voltammetry (DPV). The designs of the SERS sensor, formed on a Au(111) substrate, and the electrochemical sensor, formed on a polycrystalline Au-disk electrode (AuDE), are presented in Scheme 1. These two types of sensors had the following compositions:

(1) The SERS sensor, formed on a $\mathrm{Au}(111)$ substrate, was coated with a monolayer of magneto-plasmonic nanocarriers, bound to the substrate via a dithiolate linkage (HDT), as follows: Au(111)@HDT/MNP@MPA, where the solution side of the MNPs was coated with MPA after MNP binding to the substrate. This SERS sensor is called in this work the magneto-plasmonic nanogrid Raman sensor (MPR sensor).

(2) The electrochemical sensor, formed on a polycrystalline Au-disk electrode (AuDE) was coated with two structural layers of rGO and MNP grid, and had the composition: AuDE@CYS/rGO@PATP/MNP@MPA. The use of the rGO basal layer enabled binding of MNPs via PATP linkage and blocking the diffusion of redox probe ions $\left(\mathrm{Fe}^{2+} / \mathrm{Fe}^{3+}\right)$ to the AuDE substrate. This electrochemical sensor is called in this work the magneto-plasmonic nanogrid rGO disk electrode sensor (MPE sensor).

These two types of sensor grids have enabled not only to monitor the MNP nanocarrier functionalization but also to follow the stimulated drug release transients using solutions simulating the conditions of healthy and cancer cells.

\subsection{Assembling MPR biosensors with drug nanocarrier-mimetic NPs' grid}

Raman sensors with high SERS amplification for studies of drug nanocarriers' functionalization and anticancer drug loading and their release were designed on the basis of $\mathrm{Au}(111)$ substrates modified with a grid of NPs (20-100 nm diameter) serving as a monolayer collection of drug nanocarrier-mimetic nanoparticles. Initially, to optimize the SERS sensor design, Raman markers were applied. In Figure 1A, SERS spectrum of a Raman marker, 5-phenyl-1,3,4-oxadiazole-2-thiol (PODAT), adsorbed on a grid of GNPs bound to a $\mathrm{Au}(111)$ substrate is presented. The Raman bands at $1609.5 \mathrm{~cm}^{-1}$ and $1569 \mathrm{~cm}^{-1}$ are assigned to vibrations of $\mathrm{C}=\mathrm{N}$ and $\mathrm{N}=\mathrm{N}$ groups of the adsorbed PODAT molecules, 
respectively. This evidence indicates that the Raman marker has been successfully attached to the sensor surface using a dithiol linker and GNPs. The intensity of the scattering peak at $1609.5 \mathrm{~cm}^{-1}$ was then used for comparison with GNP grids with different NP sizes and for different dithiols used for GNP binding, as illustrated in Figure 1B for GNPs of 50 and $100 \mathrm{~nm}$ diameter and two kinds of dithiols: 3,6-dioxa-1,8-octanedithiol and 1,6-hexanedithiol. The recorded PODAT signal intensity depends on the surface area of GNPs available for Raman marker adsorption and on the plasmonic field enhancement due to the overlap of electromagnetic fields in narrow spaces between GNPs. As seen, the highest SERS intensity was obtained for $50 \mathrm{~nm}$ GNPs and the shorter linker, 1,6-hexanedithiol. Therefore, these conditions were utilized in further SERS sensor designs in this work. Similar size dependent effects have been found by Toro et al. [73] in studies of AuNP drug nanocarriers internalized in pancreas cancer cells.
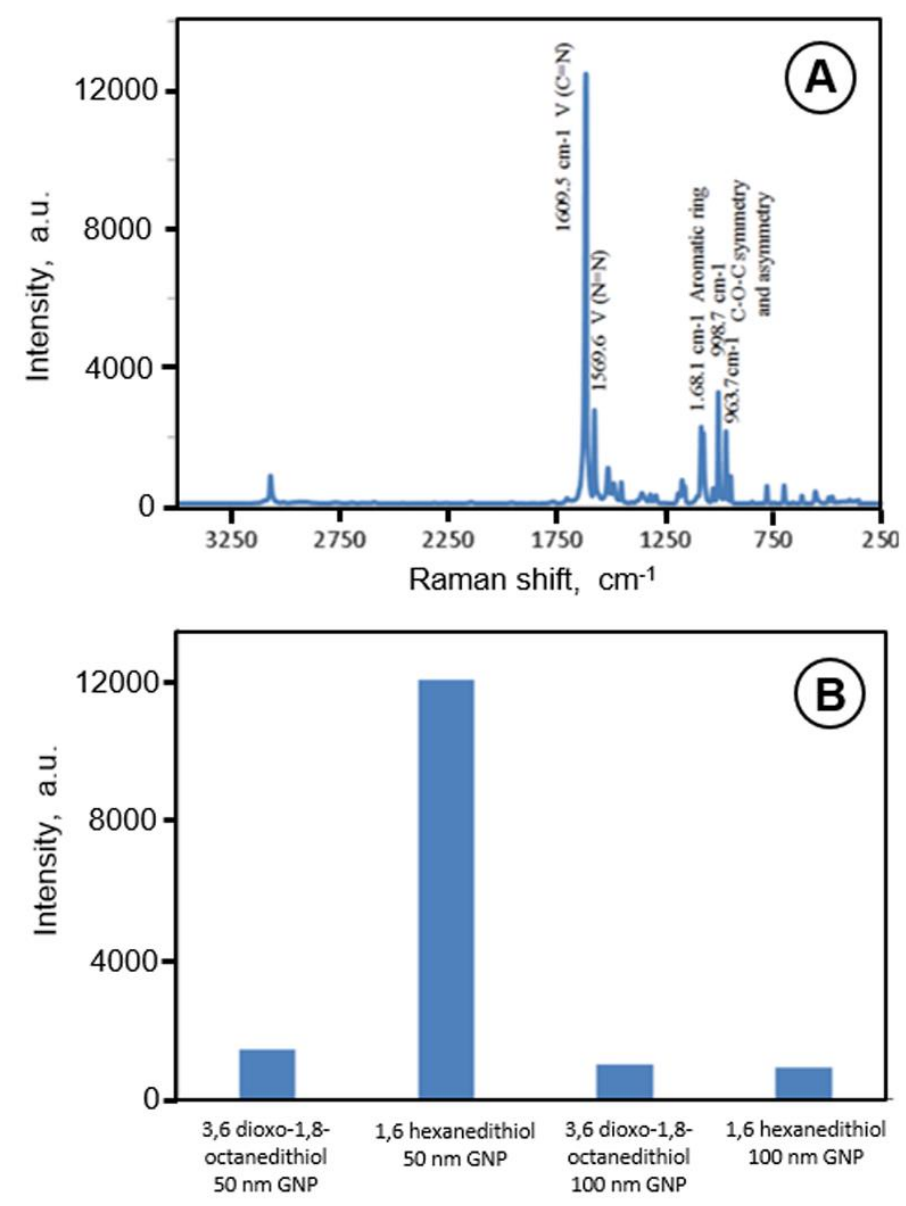

Figure 1. Testing the effects of different dithiol linkers and GNP sizes, using a Raman marker 5phenyl-1,3,4-oxadiazole-2-thiol (PODAT), to optimize the GNP grid-enhanced SERS sensor for drug nanocarrier development. (A) SERS spectrum of PODAT adsorbed on a Au(111)/GNP-grid electrode surface; the $\mathrm{Au}(111) / \mathrm{GNP}$-grid electrode was obtained here by depositing a SAM of 1,6hexanedithiol (HDT) on a $\mathrm{Au}(111)$ substrate, followed by binding GNPs (50 nm dia.) and adsorbing PODAT on the free surface of GNPs. (B) The effect of different dithiols and GNP size on the PODAT peak intensity at $1609 \mathrm{~cm}^{-1}$ : (1) 3,6-dioxa-1,8-octanedithiol (DOODT) and $50 \mathrm{~nm}$ GNPs, (2) HDT and $50 \mathrm{~nm}$ GNPs, (3) DOODT and $100 \mathrm{~nm}$ GNPs, (4) HDT and $100 \mathrm{~nm}$ GNPs.

\subsection{SERS monitoring of doxorubicin loading onto magnetic NP nanocarriers}

The progress of loading of a protected DOX (pDOX) onto a GNPs-modified SERS sensor was monitored by recording the Raman spectra associated with loaded pDOX. A typical spectrum, presented in Figure 2, revealed several characteristic peaks at $1686 \mathrm{~cm}$ 1, $1645 \mathrm{~cm}^{-1}, 1396 \mathrm{~cm}^{-1}, 978.5 \mathrm{~cm}^{-1}, 1396 \mathrm{~cm}^{-1}, 3012 \mathrm{~cm}^{-1}, 3089 \mathrm{~cm}^{-1}$ and $3246 \mathrm{~cm}^{-1}$. These 
Raman peaks are related to $\mathrm{C}=\mathrm{C}, \mathrm{C}=\mathrm{O}$, aromatic $\mathrm{C}=\mathrm{C}$, aromatic $\mathrm{C}=\mathrm{S}, \mathrm{C}-\mathrm{H}, \mathrm{OH}$ and $\mathrm{N}-\mathrm{H}$ vibrations, respectively. The resulting spectra are in excellent agreement with literature data [74-76] and the DOX structure (Scheme 2). This also means that the substrate was covered successfully with pDOX. The appearance of peaks at 1645 and $3246 \mathrm{~cm}^{-1}$, corresponding to bending vibrations of $\mathrm{CO}$ and $\mathrm{NH}$, indicates that doxorubicin is conjugated to GNP via an amide linkage to MPA (Figure 2).

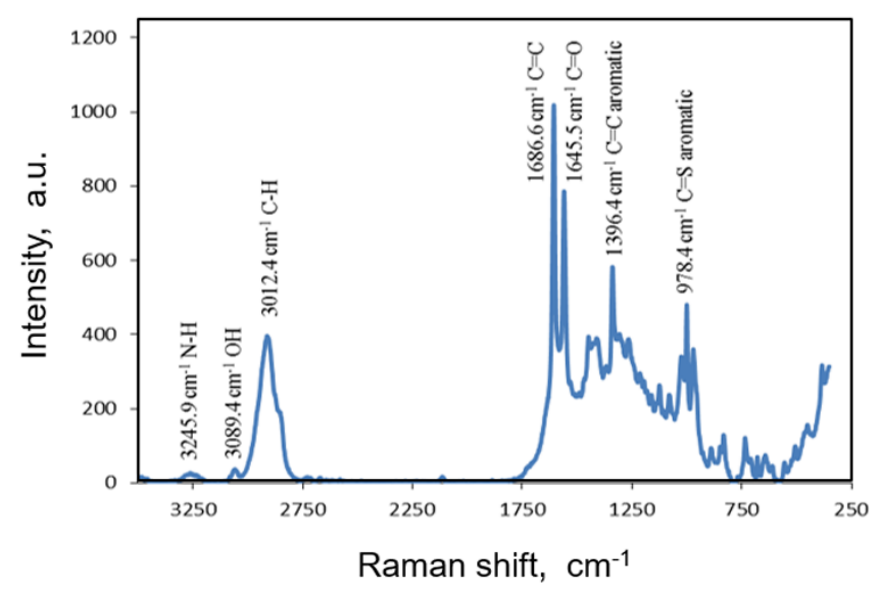

Figure 2. Raman spectrum for a protected anticancer drug doxorubicin (pDOX) bound to model GNP nanocarriers immobilized on an MPR SERS biosensor, Au(111)@HDT/GNP50nm@pDOX.

\subsection{Electrochemical monitoring of sensor functionalization}

Since the electrochemical analysis is very sensitive and can be readily employed in sensing applications [53], we have also carried out measurements using stationary and relaxation voltammetric techniques, such as the cyclic voltammetry $(\mathrm{CV})$ and differential pulse voltammetry (DPV), for characterization of MNP-modified gold probes. The use of electrochemical techniques enables monitoring and confirmation of nanocarrier modifications [77], as well as the drug loading and release. In these measurements, we have applied a redox probe $\mathrm{Fe}^{2+} / \mathrm{Fe}^{3+}$ able to penetrate surface films and provide the information about changes in film permeation due to the surface modification, drug loading, and its release.

In Figure 3A, CV characteristics for a Raman probe undergoing modifications are presented. As can be seen, a pair of peaks between $0.1 \mathrm{~V}$ and $0.3 \mathrm{~V}$ is clearly discernible in all CVs. These peaks are attributed to the redox processes of $\mathrm{Fe}^{2+} / \mathrm{Fe}^{3+}$ couple. The redox peak currents increase from the original height for a bare electrode (curve 1), to that for a modified electrode (curve 2), upon the electrode functionalization with a reduced graphene oxide film, AuDE/CYS/rGO. Further increase of peak currents is observed after binding GNPs on top of rGO (curve 3).

Similar experiments were also performed using differential pulse technique (Figure 3B). The same trend of the peak current increase upon functionalization of the probe with rGO and GNPs was observed. However, due to the better discrimination against capacitive current, the analytical signal of DPV can be determined with higher precision.

These experiments confirm suitability of the electrochemical techniques for monitoring of the Raman probes' functionalization, including the deposition of MNP and GNP grids and graphene oxide films. 

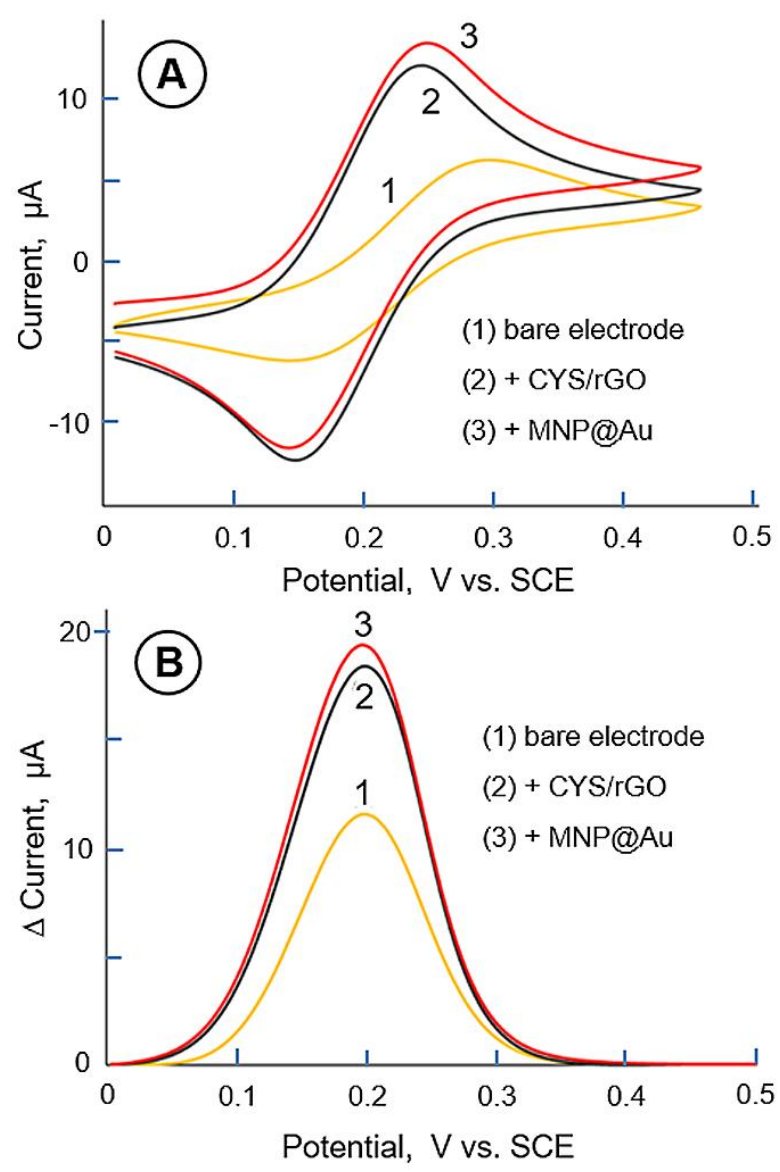

Figure 3. (A) Cyclic voltammograms for $\mathrm{Fe}^{2+} / \mathrm{Fe}^{3+}$ couple recorded using: (1) bare AuDE electrode, (2) AuDE/CYS/rGO, (3) AuDE/CYS/rGO/Fe2Ni@Au MPE sensor; scan rate $v=100 \mathrm{mV} / \mathrm{s}$. (B) Differential pulse voltammograms for $\mathrm{Fe}^{2+} / \mathrm{Fe}^{3+}$ on: (1) bare AuDE electrode, (2) AuDE/CYS/rGO, (3) $\mathrm{AuDE} / \mathrm{CYS} / \mathrm{rGO} / \mathrm{Fe}_{2} \mathrm{Ni} @ \mathrm{Au} \mathrm{MPE}$ sensor; conditions: amplitude $0.05 \mathrm{~V}$, pulse width $0.05 \mathrm{~V}$.

In a similar way, electrochemical relaxation techniques were also tested for monitoring of drug loading onto the nanocarriers. Representative DPV data are shown in Figure $4 \mathrm{~B}$, first four groups of blocks. These experiments indicate that the DPV oxidation current of $\mathrm{Fe}^{2+}$ redox probe decreased upon DOX immobilization on the electrode surface, as expected, due to the increased blocking of the electrode surface, hindering the charge-transfer process, and slowing down the diffusion of redox probe ions through the NP film.

\subsection{Monitoring of $p H$-induced drug release with SERS-electrochemical sensors}

Testing of magneto-plasmonic nanocarrier grid sensors, MPR and MPE, were performed for sensors functionalized with protected anticancer drug pDOX (Figure 4), as well as for multifunctional sensors with model nanocarriers coated with pDOX, folate targeting ligand (FTL), and biocompatibility ligand DOOT (Figure 5).

The investigations of DOX release have been carried out in solutions with acidities lowered below the physiological pH 7.4 to simulate the conditions of cancer cells. In tumor tissues, a pH value of 6 or less is readily attained since during the generation of ATP by the anaerobic glucose metabolism, with lactic acid formation as a byproduct, a large number of hydrogen ions is being produced. Even lower $\mathrm{pH}$ values, in the range 3.0-5.5, are encountered within the cancer cells in acidic intracellular organelles, such as the endosomes and lysosomes [26]. Hence, in the simulation of cancer cell conditions, the drug release experiments were performed at $\mathrm{pH} 5.3$ and 4.5 (Figure 4). The CVs and DPVs were obtained for 2, 4, 6 and 24 hours of DOX release time. 
In Figure 4B, the anodic peak currents observed in DPV experiments upon the amide bond acidolysis and DOX release process are shown. The obtained results indicate that the drug release efficacy is the highest during the first 2 hours of the interaction between the model MNP@pDOX nanocarriers immobilized on an MPE sensor and solutions, with faster release observed for $\mathrm{pH} 4.5$, as expected. During the first 2 hours, $46.2 \pm 2.3 \%$ of DOX was released and around $65.4 \pm 2.5 \%$ of the drug was released during the first 4 hours of release. After $24 \mathrm{~h}$, the DOX release amounted to $84.0 \pm 3.1 \%$.
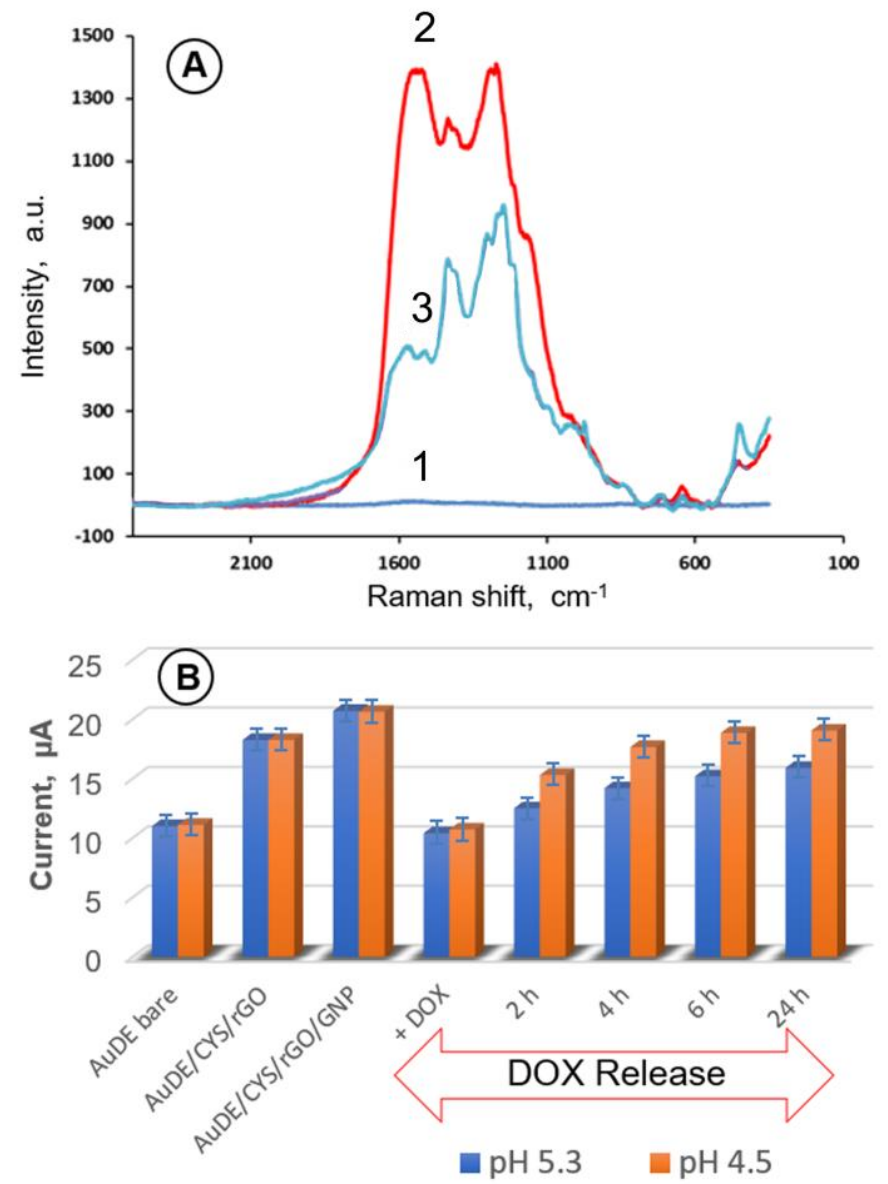

Figure 4. (A) Raman spectra for a protected anticancer drug doxorubicin (pDOX) adsorbed on model magneto-plasmonic nanocarriers ( $\mathrm{Fe}_{2} \mathrm{Ni} @ \mathrm{Au}$ ), immobilized on an MPR sensor: (1) blank sensor without any modification, (2) after incubation with pDOX, (3) after $4 \mathrm{~h}$ of drug release (pH 5.3). (B) DPV peak currents for the redox probe $\mathrm{Fe}^{2+} / \mathrm{Fe}^{3+}$ at a AuDE@CYS/rGO/MNP/pDOX electrode during the DOX release time, up to $24 \mathrm{~h}$, at pH 5.3 (blue columns) and 4.5 (red columns).

The release of drugs from $\mathrm{pH}$-responsive nanocarriers is here due to the acidolysis of amide bonds. The mechanism of this process has been thoroughly studied by Szostak et al. [78] and recently discussed by Running et al. [42] for amide-bonded dabrafenib utilized in nanocarrier-based drug delivery system for melanoma treatment. The effects of electron withdrawing properties of acid and amine substituents in asymmetric amides and amide bond twisting were indicated as amide bond weakening factors of this otherwise stable binding. Thus, amides may hydrolyze in aqueous acidic solutions $(\mathrm{pH} \leq 6)$ releasing to the solution the amine fragment (DOX) [69].

\subsection{Doxorubicin delivery using pegylated nanocarriers with targeting folate ligands}

To achieve targeted delivery, the surface of MNPs was further modified with folic acid (FA). The MNPs functionalized with thiolated folate ligands (FTL) in their shells can recognize folate-receptors overexpressed in membranes of cancer cells. Binding of MNPs 
by FA-receptors is followed by endocytosis and induced drug release in cytosol. The ligands improving nanocarrier's biocompatibility were also attached to the MNP surface. We have found it convenient to utilize 3,6-dioxa-octanethiol (DOOT) for this purpose. The drug release from MNP@(pDOX,FTL,DOOT) nanocarriers was investigated using SERS in solutions with $\mathrm{pH}$ simulating conditions of cancer cells.

As can be seen in Figure 5, the drug release is not linear in time. At $\mathrm{pH} 4.5, \mathrm{ca}$. $64.2 \pm 2.5 \%$ of loaded DOX was released from MNP nanocarriers during the first $4 \mathrm{~h}$, and $77.5 \pm 3.1 \%$ of loaded DOX was released in a $12 \mathrm{~h}$ period. These results indicate that the DOX release from the model nanocarriers functionalized with FTL and DOOT is lower than that in absence of FTL and DOOT, but the decrease is only within ca. $6.5 \%$ range.
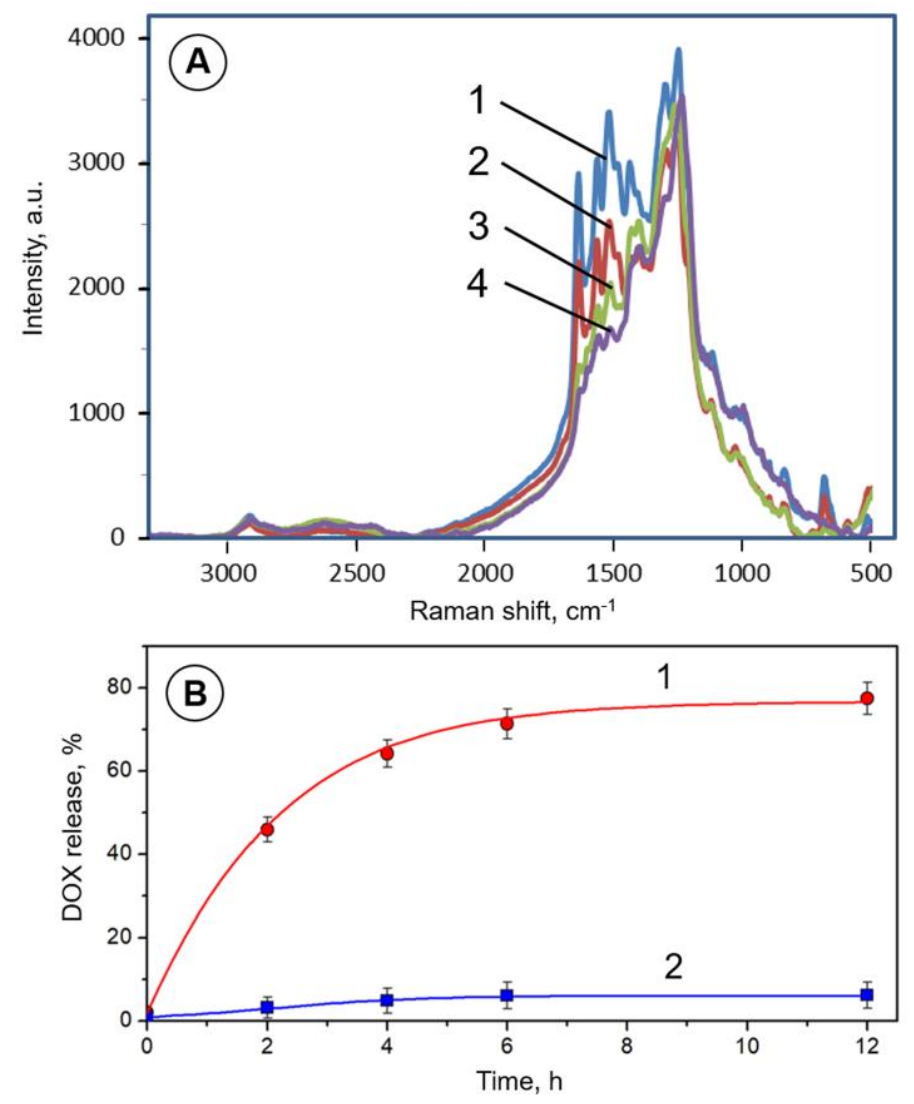

Figure 5. (A) Raman spectra of an MPR sensor with a monolayer of MNPs ( $\mathrm{Fe}_{2} \mathrm{Ni} @ \mathrm{Au}$ ) modified with protected doxorubicin (pDOX), thiolated folate ligand (FTL), and biocompatibility ligand DOOT, recorded after drug release time $t_{\text {rel }}$ [h]: (1) $0 \mathrm{~h}$; (2) $2 \mathrm{~h}$; (3) $4 \mathrm{~h}$; (4) $6 \mathrm{~h}$, under simulated cancer cell conditions with $\mathrm{pH}=5.3$. (B) Temporal evolution of DOX release from MNP nanocarriers determined from changes of the intensity of Raman scattering peak of DOX at $1651 \mathrm{~cm}^{-1}$ for: (1) $\mathrm{pH}$ 4.5 ; (2) $\mathrm{pH} 7.4$.

\section{Conclusions}

A new nanoparticle-grid based biosensing platform for developing drug nanocarriers for targeted delivery systems has been designed and tested. The proposed sensor grid, consisting of a monolayer of model magnetic gold-coated core-shell Fe2Ni@Au nanoparticles, was functionalized with folic acid targeting ligand, model protected chemotherapeutic drug doxorubicin (pDOX), and a biocompatibility agent, 3,6,-dioxa-octanethiol (DOOT). The dual signal transduction based on electrochemical and enhanced Raman scattering detection, employed in the proposed sensor, enabled convenient monitoring of DOX loading onto the nanocarriers and its releasing under simulated intracellular conditions. We have demonstrated a $\mathrm{pH}$-dependent release of DOX from drug nanocarriers immobilized on the sensor surface, under intracellular low-pH conditions of cancer cells. 
The enhanced sensitivity of Raman scattering was achieved owing to the overlap of the plasmonic fields emanating from the Au-shells of the model drug nanocarriers, creating "hot-spots" for Raman signals' amplification. The proposed nanocarrier grid devices can serve for designing and optimization of novel drug nanocarriers for new safe TDD systems, studied to mitigate adverse side effects and high collateral damage to non-tumorigenic cells encountered in classical chemotherapy with systemic drug administration.

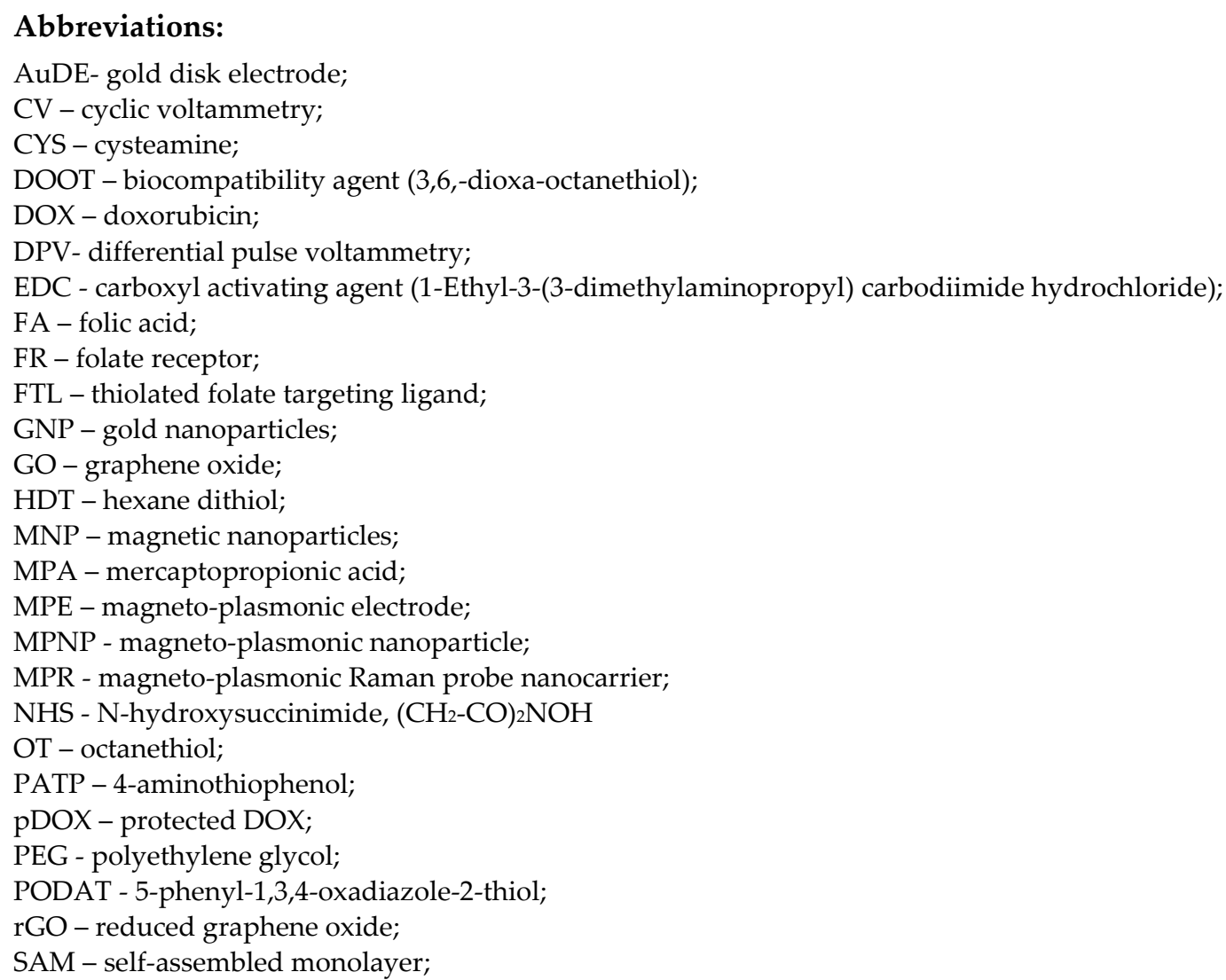

Author Contributions: Conceptualization, M.H.; methodology, H.I.; validation, H.I., M.H.; formal analysis, H.I.; investigation, H.L, C.J.Z; resources, C.J.Z, M.H.; data curation, H.I.; writing - original draft preparation, H.I.; writing - review and editing, M.H.; visualization, H.I.; supervision, C.J.Z, M.H.; project administration, M.H.; funding acquisition, C.J.Z., M.H. All authors have read and agreed to the published version of the manuscript.

Funding: This research was funded by the U.S.A. National Science Foundation, grant number DUECCLI \#0941364.

Data Availability Statement: Data are contained within the article.

Conflicts of Interest: The authors declare no conflict of interest.

\section{References}

1. Allen, T.M. Ligand-targeted therapeutics in anticancer therapy. Nat. Rev. Drug Discov. 2002, 2, 750-763.

2. Ferrari, M. Cancer nanotechnology: opportunities and challenges. Nat. Rev. Cancer 2005, 5, 161-171; doi: 10.1038/nrc1566.

3. Nguyen, N.T.; Shaegh, S.A.M.; Kashaninejad, N.; Phan, D.T. Design, fabrication and characterization of drug delivery systems based on lab-on-a-chip technology. Adv. Drug Deliv. Rev. 2013, 65, 1403-1419; doi: http://dx.doi.org/10.1016/j.addr.2013.05.008. 
4. Wang, H.; Zheng, L.; Peng, C.; Shen, M.; Shi, X.; Zhang, G. Folic acid-modified dendrimer-entrapped gold nanoparticles as nanoprobes for targeted CT imaging of human lung adenocarcinoma. Biomaterials 2013, 34, 470-480; doi: http://dx.doi.org/10.1016/j.biomaterials.2012.09.054.

5. Park, H.; Tsutsumi, H.; Mihara, H. Cell-selective intracellular drug delivery using doxorubicin and $\alpha$-helical peptides conjugated to gold nanoparticles. Biomaterials 2014, 35, 3480-3487; doi: http://dx.doi.org/10.1016/j.biomaterials.2013.12.094.

6. Patel, N.R.; Pattni, B.S.; Abouzeid, H.; Torchilin, V.P. Nanopreparations to overcome multidrug resistance in cancer. Adv. Drug Delivery Rev. 2013, 65, 1748-1762; doi: 10.1016/j.addr.2013.08.004.

7. Malam, Y.; Loizidou, M.; Seifalian, A.M. Liposomes and nanoparticles: nanosized vehicles for drug delivery in cancer. Trends Pharmacol. Sci. 2009, 30, 592-599; doi: 10.1016/j.tips.2009.08.004.

8. Manjunath, K.; Venkateswarlu, V. Pharmacokinetics, tissue distribution and bioavailability of clozapine solid lipid nanoparticles after intravenous and intraduodenal administration. J. Control. Release 2005, 107, 215-228; doi: 10.1016/j.jconrel.2005.06.006.

9. Kurzatkowska, K.; Pazos, M.A.; Herschkowitz, J.I.; Hepel, M. Cancer-Targeted Controlled Delivery of Chemotherapeutic Anthracycline Derivatives Using Apoferritin Nanocage Carriers. Int. J. Molec. Sci. 2021, 22, 1262; doi: 10.3390/ijms22031362.

10. Kowalczyk, A.; Kasprzak, A.; Poplawska, M.; Ruzycka, M.; Grudzinski, I.P.; Nowicka, A.M. Controlled drug release and cytotoxicity studies of beta-lapachon and doxorubicin loaded into cyclodextrins attached to a polyethyleneimine matrix. Int. J. Molec. Sci. 2020, 21, 5832; doi: 10.3390/ijms21165832.

11. Ganeshkumar, M.; Ponrasu, T.; Raja, M.D.; Subamekala, M.K.; Suguna, L. Green synthesis of pullulan stabilized gold nanoparticles for cancer targeted drug delivery. Spectrochimica Acta Part A: Molecular and Biomolecular Spectroscopy 2014, 130, 64-71; doi: http://dx.doi.org/10.1016/j.saa.2014.03.097.

12. Kurzatkowska, K.; Santiago, T.; Hepel, M. Plasmonic nanocarrier grid-enhanced Raman sensor for studies of anticancer drug delivery. Biosens. Bioelectron. 2017, 91, 780-787.

13. Santiago, T.; DeVaux, R.S.; Kurzatkowska, K.; Espinal, R.; Herschkowitz, J.I.; Hepel, M. Surface-enhanced Raman scattering investigation of targeted delivery and controlled release of gemcitabine. Int. J. Nanomed. 2017, 12, 7763-7776; doi: 10.2147/ijn.s149306.

14. Hepel, M. Magnetic nanoparticles for nanomedicine. Magnetochem. 2020, 6, 3; doi: 10.3390/magnetochemistry6010003.

15. Stobiecka, M.; Hepel, M. Effect of buried potential barrier in label-less electrochemical immunodetection of glutathione and glutathione-capped gold nanoparticles. Biosens. Bioelectron. 2011, 26, 3524-3530.

16. Stobiecka, M.; Hepel, M. Double-shell gold nanoparticle-based DNA-carriers with poly-L-lysine binding surface. Biomaterials 2011, 32, 3312-3321.

17. Ilkhani, H.; Arvand, M.; Ganjali, M.R.; Marrazza, G.; Mascini, M. Nanostructured Screen Printed Graphite Electrode for the Development of a Novel Electrochemical Genosensor. Electroanalysis 2013, 25, 507-514; doi: 10.1002/elan.201200556.

18. Ilkhani, H.; Hughes, T.; Li, J.; Zhong, C.J.; Hepel, M. Nanostructured SERS-electrochemical biosensors for testing of anticancer drug interactions with DNA. Biosens. Bioelectron. 2016, 80, 257-264.

19. Nowicka, A.M.; Kowalczyk, A.; Stojek, Z.; Hepel, M. Nanogravimetric and voltammetric DNA-hybridization biosensors for studies of DNA damage by common toxicants and pollutants. Biophys. Chem. 2010, 146, 42-53.

20. Kubesa, O.; Morrisey, K.; Matthews, S.; Proetta, J.; Li, C.; Skladal, P.; Hepel, M. Design of Novel Biosensors for Determination of Phenolic Compounds using Catalyst-Loaded Reduced Graphene Oxide Electrodes. Mediterr. J. Chem. 2014, 3, 916-928.

21. Stobiecka, M.; Hepel, M. Rapid functionalization of metal nanoparticles by moderator-tunable ligand-exchange process for biosensor designs. Sensors Actuators B 2010, 149, 373-380.

22. Hepel, M.; Stobiecka, M. Supramolecular interactions of oxidative stress biomarker glutathione with fluorone black. Spectrochim. Acta A 2018, 192, 146-152. 
23. Heidel, J.D.; Davis, M.E. Clinical developments in nanotechnology for cancer therapy. Pharm. Res. 2011, 28, 187-199; doi: 10.1007/s11095-010-0178-7.

24. Vigderman, L.; Zubarev, E.R. Therapeutic platforms based on gold nanoparticles and their covalent conjugates with drug molecules. Adv. Drug Deliv. Rev. 2013, 65, 663-676; doi: http://dx.doi.org/10.1016/j.addr.2012.05.004.

25. Papasani, M.R.; Wang, G.; Hill, R.A. Gold nanoparticles: the importance of physiological principles to devise strategies for targeted drug delivery. Nanomedicine: Nanotechnol. Biol. Med. 2012, 8, 804-814; doi: http://dx.doi.org/10.1016/j.nano.2012.01.008.

26. Tiğli Aydin, R.S.; Pulat, M. 5-fluorouracil encapsulated chitosan nanoparticles for pH-stimulated drug delivery: Evaluation of controlled release kinetics. J. Nanomater. 2012, 2012; doi: 10.1155/2012/313961.

27. Kushwaha, S.K.S.; Rastogi, A.; Rai, A.K.; Singh, S. Novel drug delivery system for anticancer drug: A review. Int. J. Pharm. Res. 2012, 4, 542-553.

28. Lu, F.; Doane, T.L.; Zhu, J.J.; Burda, C. Gold nanoparticles for diagnostic sensing and therapy. Inorg. Chim. Acta 2012, 393, 142-153; doi: http://dx.doi.org/10.1016/j.ica.2012.05.038.

29. Bunker, A. Poly(ethylene glycol) in drug delivery, why does it work, and can we do better? All atom molecular dynamics simulation provides some answers. Physics Procedia 2012, 34, 24-33; doi: 10.1016/j.phpro.2012.05.004.

30. Hepel, T. Functional Nanoparticle-Based Bioelectronic Devices. In Functional Nanoparticles for Bioanalysis, Nanomedicine and Bioelectronic Devices, Vol. 2, Hepel, M., Zhong, C.J., Eds. Oxford University Press: Oxford, 2012; Vol. 2; doi: 10.1021/bk-20121113.ch008.

31. Bevilacqua, P.; Nuzzo, S.; Torino, E.; Condorelli, G.; Salvatore, M.; Grimaldi, A.M. Antifouling Strategies of Nanoparticles for Diagnostic and Therapeutic Application: A Systematic Review of the Literature. Nanomaterials 2021, 11, 780; doi: 10.3390/nano11030780.

32. Gajbhiye, K.R.; Pawar, A.; Mahadik, K.R.; V.Gajbhiye. PEGylated nanocarriers: A promising tool for targeted delivery to the brain. Colloids Surf. B: Biointerf. 2020, 187, 110770; doi: 10.1016/j.colsurfb.2019.110770.

33. Vilaça, N.; Amorim, R.; Machado, A.F.; Parpot, P.; Pereira, M.F.R.; Sardo, M.; Rocha, J.; Fonseca, A.M.; Neves, I.C.; Baltazar, F. Potentiation of 5-fluorouracil encapsulated in zeolites as drug delivery systems for in vitro models of colorectal carcinoma. Colloids Surf. B: Biointerf. 2013, 112, 237-244; doi: 10.1016/j.colsurfb.2013.07.042.

34. Dharmatti, R.; Phadke, C.; Mewada, A.; Pandey, S.; Oza, G.; Sharon, C.; Sharon, M. Surface Orchestration of Gold Nanoparticles Using Cysteamine as Linker and Folate as Navigating Molecule for Synaphic Delivery of doxorubicin. J. Nanomed. Res. 2014, 1; doi: 10.15406/jnmr.2014.01.00002.

35. Sahu, S.K.; Maiti, S.; Pramanik, A.; Ghosh, S.K.; Pramanik, P. Controlling the thickness of polymeric shell on magnetic nanoparticles loaded with doxorubicin for targeted delivery and MRI contrast agent. Carbohydrate Polymers 2012, 87, 25932604; doi: 10.1016/j.carbpol.2011.11.033.

36. Lu, B.; Xiong, S.B.; Yang, H.; Yin, X.D.; Chao, R.B. Solid lipid nanoparticles of mitoxantrone for local injection against breast cancer and its lymph node metastases. Eur. J. Pharm. Sci. 2006, 28, 86-95; doi: 10.1016/j.ejps.2006.01.001.

37. Zhang, T.; Li, G.; Guo, L.; Chen, H. Synthesis of thermo-sensitive CS-g-PNIPAM/CMC complex nanoparticles for controlled release of 5-FU. Int. J. Biol. Macromolec. 2012, 51, 1109-1115; doi: http://dx.doi.org/10.1016/j.ijbiomac.2012.08.033.

38. Bhattacharya, R.; Patra, C.R.; Earl, A.; Wang, S.; Katarya, A.; Lu, L.; Kizhakkedathu, J.N.; Yaszemski, M.J.; Greipp, P.R.; Mukhopadhyay, D., et al. Attaching folic acid on gold nanoparticles using noncovalent interaction via different polyethylene glycol backbones and targeting of cancer cells. Nanomedicine: Nanotechnol. Biol. Med. 2007, 3, 224-238; doi: http://dx.doi.org/10.1016/j.nano.2007.07.001.

39. Zhang, Z.; Jia, J.; Lai, Y.; Ma, Y.; Weng, J.; Sun, L. Conjugating folic acid to gold nanoparticles through glutathione for targeting and detecting cancer cells. Bioorg Med Chem 2010, 18, 5528-5534; doi: http://dx.doi.org/10.1016/j.bmc.2010.06.045. 
40. Gunduz, U.; Keskin, T.; Tansık, G.; Mutlu, P.; Yalcın, S.; Unsoy, G.; Yakar, A.; Khodadust, R.; Gunduz, G. Idarubicin-loaded folic acid conjugated magnetic nanoparticles as a targetable drug delivery system for breast cancer. Biomed. Pharmacother. 2014, 68, 729-736; doi: http://dx.doi.org/10.1016/j.biopha.2014.08.013.

41. Li, J.; Skeete, Z.; Shan, S.; Yan, S.; Kurzatkowska, K.; Zhao, W.; Ngo, Q.M.; Holubovska, P.; Luo, J.; Hepel, M., et al. Surface Enhanced Raman Scattering Detection of Cancer Biomarkers with Bifunctional Nanocomposite Probes. Anal. Chem. 2015, 87, 10698-10702; doi: 10.1021/acs.analchem.5b03456.

42. Running, L.; Espinal, R.; Hepel, M. Controlled release of targeted chemotherapeutic drug dabrafenib for melanoma cancers monitored using surface-enhanced Raman scattering (SERS) spectroscopy. Mediterr. J. Chem. 2018, 7, 18-27.

43. Smith, M.; Hepel, M. Controlled release of targeted anti-leukemia drugs azacitidine and decitabine monitored using surfaceenhanced Raman scattering (SERS) spectroscopy. Mediterr. J. Chem. 2017, 6, 125-132; doi: 10.13171/mjc64/01706081223-hepel.

44. Breitkreitz, M.C.; Sabin, G.P.; Polla, G.; Poppi, R.J. Characterization of semi-solid Self-Emulsifying Drug Delivery Systems (SEDDS) of atorvastatin calcium by Raman image spectroscopy and chemometrics. J. Pharm. Biomed. Anal. 2013, 73, 3-12; doi: http://dx.doi.org/10.1016/j.jpba.2012.03.054.

45. Cipriani, P.; Smith, C.Y. Characterization of Thalidomide using Raman Spectroscopy. Spectrochim. Acta Part A: Molec. Biomolec. Spectrosc. 2008, 69, 333-337; doi: http://dx.doi.org/10.1016/j.saa.2007.04.002.

46. Armstrong, C.L.; Edwards, H.G.M.; Farwell, D.W.; Williams, A.C. Fourier transform Raman microscopic study of drug distribution in a transdermal drug delivery device. Vibrational Spectroscopy 1996, 11, 105-113; doi: http://dx.doi.org/10.1016/0924-2031(95)00066-6.

47. Davies, M.C.; Binns, J.S.; Melia, C.D.; Bourgeois, D. Fourier transform Raman spectroscopy of polymeric biomaterials and drug delivery systems. Spectrochim. Acta Part A: Molec. Spectrosc. 1990, 46, 277-283; doi: http://dx.doi.org/10.1016/05848539(90)80095-G.

48. Mariappan, G.; Sundaraganesan, N. FT-IR, FT-Raman, NMR spectra, density functional computations of the vibrational assignments (for monomer and dimer) and molecular geometry of anticancer drug 7-amino-2-methylchromone. J. Molec. Struct. 2014, 1063, 192-202; doi: http://dx.doi.org/10.1016/j.molstruc.2014.01.064.

49. Gotter, B.; Faubel, W.; Heißler, S.; Hein, J.; Neubert, R.H.H. Determination of drug content in semisolid formulations by non-invasive spectroscopic methods: FTIR - ATR, - PAS, - Raman and PDS. J. Phys.: Conf. Ser. 2010, 214, 012129; doi: 10.1088/1742-6596/214/1/012129.

50. Hargreaves, M.D.; Macleod, N.A.; Smith, M.R.; Andrews, D.; Hammond, S.V.; Matousek, P. Characterisation of transmission Raman spectroscopy for rapid quantitative analysis of intact multi-component pharmaceutical capsules. $J$. Pharm. Biomed. Anal. 2011, 54, 463-468; doi: 10.1016/j.jpba.2010.09.015.

51. Stillhart, C.; Kuentz, M. Comparison of high-resolution ultrasonic resonator technology and Raman spectroscopy as novel process analytical tools for drug quantification in self-emulsifying drug delivery systems. J. Pharm. Biomed. Anal. 2012, 59, 29-37; doi: http://dx.doi.org/10.1016/j.jpba.2011.10.018.

52. Li, Y.-S.; Church, J.S. Raman spectroscopy in the analysis of food and pharmaceutical nanomaterials. J. Food Drug Anal. 2014, 22, 29-48; doi: http://dx.doi.org/10.1016/j.jfda.2014.01.003.

53. Hepel, M.; Stobiecka, M. Detection of Oxidative Stress Biomarkers Using Functional Gold Nanoparticles. In Fine Particles in Medicine and Pharmacy, Matijevic, E., Ed. Springer Sci Publ.: New York, 2012; pp. 241-281.

54. Ding, S.Y.; Yi, J.; Li, J.F.; Ren, B.; Wu, D.Y.; Panneerselvam, R.; Tian, Z.Q. Nanostructure-based plasmon-enhanced Raman spectroscopy for surface analysis of materials. Nat. Rev. Mater. 2016, 1, 16021; doi: 10.1038/natrevmats.2016.21.

55. Willets, K.A.; Duyne, R.P.V. Localized surface plasmon resonance spectroscopy and sensing. Annu. Rev. Phys. Chem. 2007, 58, 267-297; doi: 10.1146/annurev.physchem.58.032806.104607. 
56. Zha, Z.; Liu, R.; Yang, W.; Li, C.; Gao, J.; Shafi, M.; Fan, X.; Li, Z.; Du, X.; Jiang, S. Surface-enhanced Raman scattering by the composite structure of $\mathrm{Ag}$ NP-multilayer $\mathrm{Au}$ films separated by $\mathrm{Al}_{2} \mathrm{O}_{3}$. Optics Express 2021, 29, 8890-8901; doi: 10.1364/OE.419133.

57. Kalachyova, Y.; Mares, D.; Jerabek, V.; Zaruba, K.; Ulbrich, P.; Lapchak, L.; Svorcik, V.; Lyutakov, O. The effect of silver grating and nanoparticle grafting for LSP-SPP coupling and SERS response intensification. J. Phys. Chem. C 2016, 120, 1056910577; doi: 10.1021/acs.jpcc.6b01587.

58. Hao, R.; You, H.; Zhu, J.; Chen, T.; Fang, J. “Burning Lamp”-like Robust Molecular Enrichment for Ultrasensitive Plasmonic Nanosensors. ACS Sens. 2020, 5, 781-788; doi: 10.1021/acssensors.9b02423.

59. Castro-Grijalba, A.; Montes-García, V.; Cordero-Ferradás, M.J.; Coronado, E.; Pérez-Juste, J.; Pastoriza-Santos, I. SERS-Based Molecularly Imprinted Plasmonic Sensor for Highly Sensitive PAH Detection. ACS Sens. 2020, 5, 693-702; doi: 10.1021/acssensors.9b01882.

60. Paul, W.; Sharma, C.P. Inorganic nanoparticles for targeted drug delivery. In Biointegration of Medical Implant Materials, Sharma, C.P., Ed. Woodhead Publ.: 2010; 10.1533/9781845699802.2.204pp. 204-235; ISBN: 978-1-84569-509-5; doi: 10.1533/9781845699802.2.204.

61. Arruebo, M.; Fernández-Pacheco, R.; Ibarra, M.R.; Santamaría, J. Magnetic nanoparticles for drug delivery. Nano Today 2007, 2, 22-32; doi: 10.1016/s1748-0132(07)70084-1.

62. Arias, J.L. Novel strategies to improve the anticancer action of 5-fluorouracil by using drug delivery systems. Molecules 2008, 13, 2340-2369; doi: 10.3390/molecules13102340.

63. Kouassi, G.K.; Irudayaraj, J. Magnetic and Gold-Coated Magnetic Nanoparticles as a DNA Sensor. Anal. Chem. 2006, 78, 3234-3241.

64. Wagstaff, A.J.; Brown, S.D.; Holden, M.R.; Craig, G.E.; Plumb, J.A.; Brown, R.E.; Schreiter, N.; Chrzanowski, W.; Wheate, N.J. Cisplatin drug delivery using gold-coated iron oxide nanoparticles for enhanced tumour targeting with external magnetic fields. Inorg. Chim. Acta 2012, 393, 328-333; doi: http://dx.doi.org/10.1016/j.ica.2012.05.012.

65. Mahmoudi, M.; Sahraian, M.A.; Shokrgozar, M.A.; Laurent, S. Superparamagnetic iron oxide nanoparticles: Promises for diagnosis and treatment of multiple sclerosis. ACS Chem. Neurosci. 2011, 2, 118-140; doi: 10.1021/cn100100e.

66. Robinson, I.; Tung le, D.; Maenosono, S.; Walti, C.; Thanh, N.T. Synthesis of core-shell gold coated magnetic nanoparticles and their interaction with thiolated DNA. Nanoscale 2010, 2, 2624-2630; doi: 10.1039/c0nr00621a.

67. Liu, Y.; Chi, Y.; Shan, S.; Yin, J.; Luo, J.; Zhong, C.J. Characterization of magnetic NiFe nanoparticles with controlled bimetallic composition. J. Alloy. Compd. 2014, 587, 260-266; doi: 10.1016/j.jallcom.2013.10.203.

68. Xiao, T.; Cao, X.; Shi, X. Dendrimer-entrapped gold nanoparticles modified with folic acid for targeted gene delivery applications. J. Control. Release 2013, 172, e114-e115; doi: http://dx.doi.org/10.1016/j.jconrel.2013.08.275.

69. DeRuiter, J. Amides and related funcional groups In Principles of Drug Action, 2005; Vol. 1, pp. 1-16.

70. Sundaram, P.; Wower, J.; Byrne, M.E. A nanoscale drug delivery carrier using nucleic acid aptamers for extended release of therapeutic. Nanomedicine: Nanotechnol. Biol. Med. 2012, 8, 1143-1151; doi: http://dx.doi.org/10.1016/j.nano.2012.01.010.

71. Cho, C.-S.; Kobayashi, A.; Takei, R.; Ishihara, T.; Maruyama, A.; Akaike, T. Receptor-mediated cell modulator delivery to hepatocyte using nanoparticles coated with carbohydrate-carrying polymers. Biomaterials 2000, 22, 45-51; doi: http://dx.doi.org/10.1016/S0142-9612(00)00143-5.

72. Wang, Z.; Zhou, X.; Zhang, J.; Boey, F.; Zhang, H. Direct Electrochemical Reduction of Single-Layer Graphene Oxide and Subsequent Functionalization with Glucose Oxidase. J. Phys. Chem. C 2009, 113, 14071-14075; doi: 10.1021/jp906348x.

73. Trono, J.D.; Mizuno, K.; Yusa, N.; Matsukawa, T.; Yokoyama, K.; Uesaka, M. Size, concentration and incubation time dependence of gold nanoparticle uptake into pancreas cancer cells and its future application to X-ray drug delivery system. J. Rad. Res. 2011, 52, 103-109; doi: 10.1269/jrr.10068. 
74. Stanicová, J.; Fabriciová, G.; Chinsky, L.; Sutiak, V.; Miskovsky, P. Amantadine-DNA interaction as studied by classical and resonance Raman spectroscopy. J. Molec. Struct. 1999, 478, 129-138.

75. Fodor, S.P.A.; Rava, R.P.; Hays, T.G.; Spiro, T.G. Ultraviolet resonance Raman spectroscopy of the nucleotides with 266-, 240-, 218-, and 200-nm pulsed laser excitation. J. Am. Chem. Soc. 1985, 107, 1520-1529.

76. Lee, C.J.; Kang, J.S.; Kim, M.S.; Lee, K.P.; Lee, M.S. The Study of Doxorubicin and its Complex with DNA by SERS and UVresonance Raman Spectroscopy. Bull. Korean Chem. Soc. 2004, 25, 1211-1216.

77. Hepel, M. Functional Gold Nanoparticles for Biointerfaces. In Functional Nanoparticles for Bioanalysis, Nanomedicine E Bioelectronic Devices, Hepel, M., Zhong, C.J., Eds. Oxford University Press: Oxford, 2012; Vol. 1, pp. 147-176; ISBN: 978-084122-775-0.

78. Szostak, M.; Yao, L.; Aubé, J. Stability of Medium-Bridged Twisted Amides in Aqueous Solutions. J. Org. Chem. 2009, 74, 1869-1875; doi: http://dx.doi.org/10.1021/jo802192v. 